Case Report

\title{
Mammary Analogue Secretory Carcinoma of the Parotid Gland: A Third World Country Perspective-A Case Series
}

\author{
Huzaifah Salat, ${ }^{1}$ Ramiz Mumtaz, ${ }^{2}$ Mubasher Ikram, ${ }^{2}$ and Nasir Ud Din ${ }^{3}$ \\ ${ }^{1}$ Aga Khan University Medical College, Karachi 74800, Pakistan \\ ${ }^{2}$ Department of Otolaryngology, Aga Khan University, Karachi 74800, Pakistan \\ ${ }^{3}$ Department of Pathology \& Microbiology, Aga Khan University, Karachi 74800, Pakistan
}

Correspondence should be addressed to Huzaifah Salat; huzaifah.salat@gmail.com

Received 30 September 2015; Revised 26 November 2015; Accepted 6 December 2015

Academic Editor: Chung-Feng Hwang

Copyright (C) 2015 Huzaifah Salat et al. This is an open access article distributed under the Creative Commons Attribution License, which permits unrestricted use, distribution, and reproduction in any medium, provided the original work is properly cited.

\begin{abstract}
Mammary analogue secretory carcinoma (MASC) is a recently described pathological entity in major salivary glands, which was first described by Skálová et al. in 2010. Since then only a limited number of case reports/series have been published describing this tumor with the majority of them discussing the genetic and cytoarchitectural aspect of this tumor. Keeping this in view with the lack of clinical correlation with regard to this tumor, we present our approach to management of two such cases which, according to the best of our knowledge, are the first 2 cases presenting in the South Asian continent. Both patients were diagnosed and managed at Aga Khan University Hospital, Karachi, Pakistan.
\end{abstract}

\section{Introduction}

Mammary analogue secretory carcinoma (MASC) is a recently described pathological entity in major salivary glands, with the parotid being the most common location. This salivary gland tumor has histopathological features synonymous of both salivary acinic cell carcinoma (AciCC) and low grade cystadenocarcinoma while at the same time strongly displaying significant similarities to breast secretory carcinoma [1]. Microscopically it comprises solid and microcystic areas, with microcysts filled with colloid-like PAS positive material and appearance with low grade nuclei and granular cytoplasm [2].

WHO classification of salivary gland neoplasms is complex and consists of 10 benign and 23 malignant variants [3]. Regardless, salivary gland neoplasms are not common as literature reveals an epidemiologic prevalence of $0.4-$ 13.5 cases per 100,000 people. Among the salivary gland tumors, parotid gland tumors are rare in entity themselves and account for $12 \%$ of oropharyngeal cancers or $0-3 \%$ of all cancers affecting the body [4]. In 2010, the first reported case series by Skálová et al. consisted of 16 cases out of which 13 were of parotid origin and 3 originated from minor salivary glands [5]. Henceforth, we present 2 cases of MASC of the parotid gland which to our knowledge are the first two cases in the South Asian territory. Both patients were diagnosed and managed at Aga Khan University Hospital, Karachi, Pakistan.

\section{Case 1}

A 60-year-old man, resident of Sukkur, Sindh, and an occasional alcoholic, presented to the ENT clinic with a sevenmonth history of swelling present on the right parotid area which was initially small but increasing in size gradually. $\mathrm{He}$ had no other associated symptoms. Past medical history and family history were not significant. On examination there was a $3 \times 3 \mathrm{~cm}$ firm swelling present at the right parotid area, which was mobile in all planes. Overlying skin was not involved and no neck nodes were palpable. Rest of the ENT examination was unremarkable.

The FNAC of the swelling was done which showed epithelial neoplasm possibly of salivary gland origin. So with the provisional diagnosis of suspected malignancy of right parotid gland he underwent a right parotidectomy. Intraoperatively, the mass was $4-5 \mathrm{~cm}$, hard lesion in the superficial 


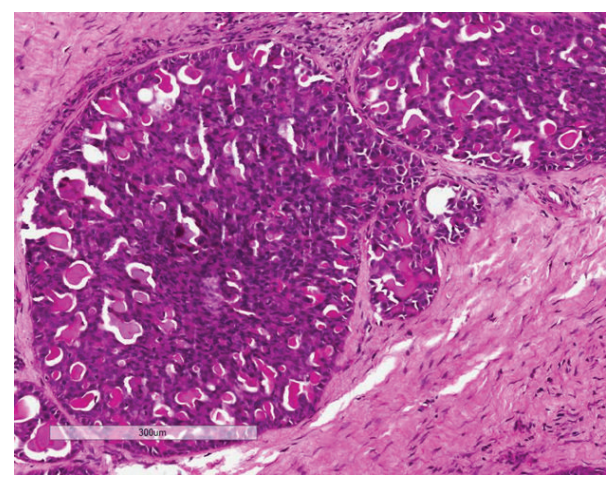

FIGURE 1: MASC displaying microcystic pattern of growth and these spaces are filled with pink colloid-like material (H\&E, 200x magnification).

lobe which was removed with clear margins while preserving the facial nerve. The final histopathology result revealed mammary analogue secretory carcinoma which was reconfirmed by four consultant histopathologists (Figure 1).

The case was then discussed in multidisciplinary tumor (MDT) meeting and it was recommended that a neck dissection should be carried out, followed by radiation therapy which was total dose of $66 \mathrm{~Gy}$ in 33 cycles. Subsequently, he underwent modified radical neck dissection on the same side followed by radiation therapy.

On follow-up after 1 month and 7 months, respectively, the patient was stable and was allowed to go back to his hometown.

\section{Case 2}

A 42-year-old male, a resident of Jacobabad, Sindh, with no known comorbid conditions and no history of addiction, visited our clinic in February 2013 with a 1-year history of swelling present in the left parotid area. He was operated on outside our institute in 2012 with the preliminary diagnosis of pleomorphic adenoma of parotid gland, but the final histopathology report revealed that there was a suspicion of malignancy with involved margins coming back positive as well. Past medical history and family history were unremarkable for this patient as well.

When we examined the mass it was $2 \times 2 \mathrm{~cm}$ left preauricular swelling which was tender, firm, and mobile. We repeated his MRI scan which showed residual mass of left parotid gland extending up to left mastoid with no nodal involvement. Subsequently, he was planned for left total parotidectomy. Intraoperatively, the lesion was found in the tail of parotid, and thus revision left total parotidectomy was done. Neck dissection was not done in this case because there were no suspicious nodes detected on radiologic scan.

Final histopathology report showed MASC (Figure 2). The case was then discussed in MDT meeting, and it was decided that the patient should receive adjuvant radiotherapy with total dose of $66 \mathrm{~Gy}$.

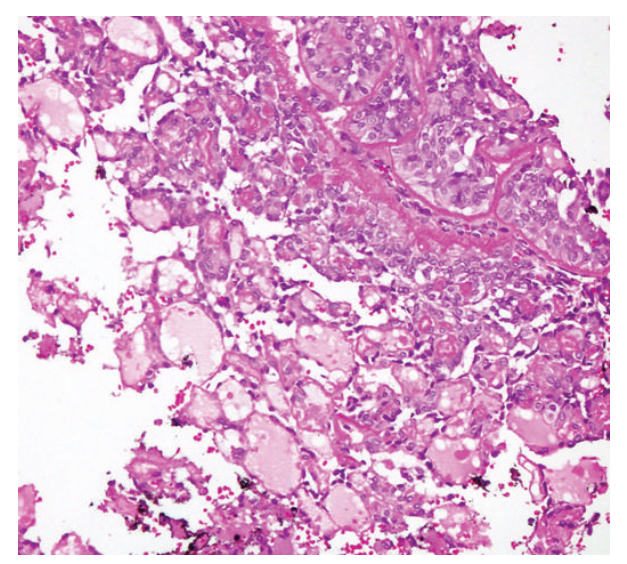

FIGURE 2: MASC displaying microcystic pattern of growth with the spaces showing pink colloid-like material.

On follow-up after 1 month and 6 months, respectively, the patient was deemed stable and was advised to come for yearly follow-up.

\section{Discussion}

In 2010, a case series comprising 16 cases was published by Skálová et al., in which a new salivary gland tumor was described and named MASC [5]. Since then, to the best of our knowledge, 92 cases [3] have been published describing this hitherto undescribed tumor, with the majority of the articles being review articles or articles discussing the genetic and cytoarchitectural aspect of this tumor. Keeping this in perspective with the lack of clinical correlation with regard to this tumor, we have highlighted our experience of management in these cases which according to our knowledge are the first two cases reported in South Asia.

Due to the considerable overlap of histological features of this tumor with other aforementioned malignancies, FNA aspirates are sometimes notable to give a conclusive answer. This was true with regard to our patient as well, as our initial FNAC result suggested a tumor of epithelial origin. However, among the 12 FNA diagnosed cases of MASC published [3], only one [6] received an initial diagnosis of MASC. The question of whether surgical biopsy is the best method of diagnosis still remains unanswered. As far as diagnosis is concerned in developing countries, extensive immunohistochemical analysis is not possible. Firstly, it is not easily available and secondly because health care in developing countries like Pakistan is through one's own pockets with no federal government or insurance companies to support, immunohistochemical analysis remains to be an academician dream. Postoperative radiotherapy (PORT) is usually done for close $(<5 \mathrm{~mm})$ margins/incomplete resection, perineural invasion, and all tumors greater than T2 size, whereas in cases of suspicion of low grade malignancy radical surgical resection is usually the standard protocol [7].

Hence, to bridge the gap between diagnostics and interventions, ancillary studies are being carried out to effectively diagnose MASC using various modalities. Genetic studies 


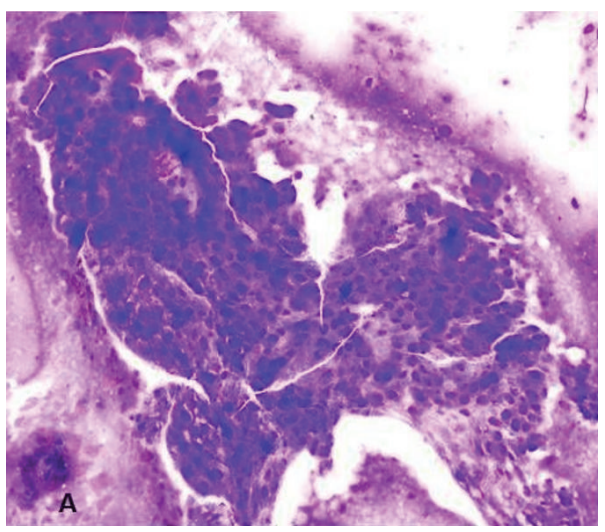

(a)

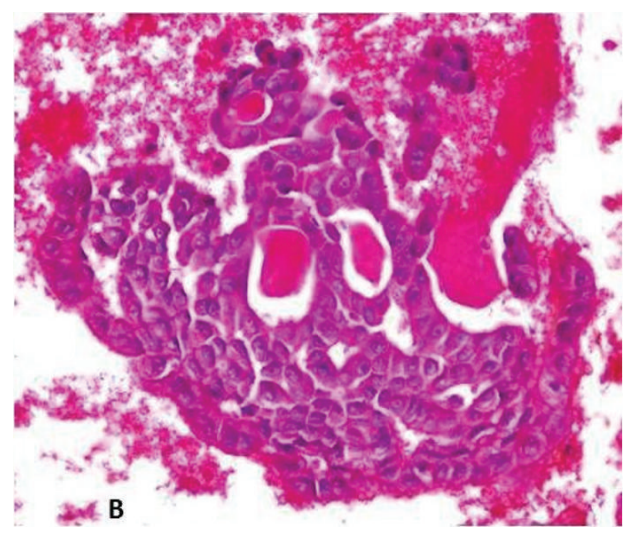

(b)

Figure 3: Cytology smears of FNAC ((a) Diff-Quick and (b) H\&E stain) show a low grade epithelial tumor with round to oval nuclei. Eosinophilic secretions are seen in H\&E image (b).

suggest that these tumors possess a chromosomal translocation $\mathrm{t}(12 ; 15)-(\mathrm{p} 13 ; \mathrm{q} 25)$ analogous to breast carcinoma, resulting in a fusion oncogene ETV6-NTRK3 [8]. This fusion results in the activation of tyrosine kinase which further potentiates the activation of Ras-MAP mitogen pathway and phosphatidylinositol 3-kinase-AKT pathway $[9,10]$. The significance of this translocation is that this was the exact translocation first detected in infantile fibrosarcoma and congenital mesoblastic nephroma $[11,12]$. In addition, similar translocations have also been consistent findings in secretory carcinoma of breast, hence the name secretory analogue carcinoma [5].

Keeping in mind the complexity and heterogeneity of presentations of salivary gland tumors and to effectively diagnose our cases, we used fine needle aspiration cytology and immunohistochemistry markers such as S100, Cytokeratin 19, EMA (epithelial membrane antigen), and DOG 1 stain. Both our cases on histology specimens confirmed low grade MASC tumor as they grew in a microcystic pattern, with eosinophilic secretions noted in the luminal spaces along with uniform nuclei (Figure 3 ). We ruled out acinic cell carcinoma (AciCC) on the basis of DOG1 stain which is negative in MASC and the fact that AciCC should at least demonstrate focal basophilic cytoplasmic granules and macronuclei [5]. We also ruled out low grade mucoepidermoid carcinoma (MEC) as our cases did not display the abundant epidermoid cells and mucinous cells frequently seen in MEC [13]. S100 and EMA markers are positive in MASC, whereas DOG1 stain is positive in majority of cases of AciCC [5]. These modalities were enough to come to a clear diagnosis of MASC and we recommend use of these markers along with histopathology to come to a diagnosis in resource limited countries, where extensive histochemical studies cannot be performed.

Treatment modalities mentioned in literature include details of 86 patients. $26 \%$ of the patients underwent neck dissections and $20 \%$ of patients were given postoperative radiotherapy (PORT), while $2 \%$ of the patients received PORT with chemotherapy. No reported patients were given radiotherapy before surgery. Due to the limited data available with regard to treatment protocols, surgeons may currently consider neck dissection as the safest intervention, especially since we cannot conclude whether MASC comes under the category of low grade malignant salivary tumors or not.

\section{Conclusion}

Published data remains unclear as to which modality is more superior over the other. Overwhelming amount of research is being done on the cytochemical aspects of MASC rather than on its diagnosis and management. Further research needs to be done in this area to understand the prognostic significance of MASC.

In South Asia, where there is a dearth of surgeons and lack of available extensive immunohistochemical diagnostic modalities, especially in the developing countries, we advise close clinical and surgical correlation with respect to the management of MASC via multidisciplinary tumor board meetings and strongly suggest that MASC be kept in the differential diagnosis in tumors arising from salivary glands.

\section{Conflict of Interests}

The authors declare that there is no conflict of interests regarding the publication of this paper.

\section{Acknowledgment}

The authors would like to gratefully acknowledge the assistance and valuable information provided by Dr. Khursheed Khan.

\section{References}

[1] A. Skálová, T. Vanecek, R. Sima et al., "Mammary analogue secretory carcinoma of salivary glands, containing the etv6ntrk3 fusion gene: a hitherto undescribed salivary gland tumor entity," The American Journal of Surgical Pathology, vol. 34, no. 5, pp. 599-608, 2010. 
[2] Mammary Analogue Secretory Carcinoma, 2013, http://www .E-immunohistochemistry.info.

[3] R. Sethi, E. Kozin, A. Remenschneider et al., "Mammary analogue secretory carcinoma: update on a new diagnosis of salivary gland malignancy," The Laryngoscope, vol. 124, no. 1, pp. 188-195, 2014.

[4] M. Horner, L. Ries, M. Krapcho et al., SEER Cancer Statistics Review, 1975-2006, National Cancer Institute, Bethesda, Md, USA, 2009.

[5] F. Petersson, D. Lian, Y. P. Chau, and B. Yan, "Mammary analogue secretory carcinoma: the first submandibular case reported including findings on fine needle aspiration cytology," Head and Neck Pathology, vol. 6, no. 1, pp. 135-139, 2012.

[6] P. Levine, K. Fried, L. D. Krevitt, B. Wang, and B. M. Wenig, "Aspiration biopsy of mammary analogue secretory carcinoma of accessory parotid gland: another diagnostic dilemma in matrix-containing tumors of the salivary glands," Diagnostic Cytopathology, vol. 42, no. 1, pp. 49-53, 2014.

[7] A. Al-Mamgani, P. van Rooij, G. M. Verduijn, C. A. Meeuwis, and P. C. Levendag, "Long-term outcomes and quality of life of 186 patients with primary parotid carcinoma treated with surgery and radiotherapy at the Daniel den hoed cancer center," International Journal of Radiation Oncology Biology Physics, vol. 84, no. 1, pp. 189-195, 2012.

[8] A. Connor, B. Perez-Ordoñez, M. Shago, A. Skálová, and I. Weinreb, "Mammary analog secretory carcinoma of salivary gland origin with the ETV6 gene rearrangement by FISH: expanded morphologic and immunohistochemical spectrum of a recently described entity," The American Journal of Surgical Pathology, vol. 36, no. 1, pp. 27-34, 2012.

[9] C. Tognon, S. R. Knezevich, D. Huntsman et al., "Expression of the ETV6-NTRK3 gene fusion as a primary event in human secretory breast carcinoma," Cancer Cell, vol. 2, no. 5, pp. 367376, 2002.

[10] D. H. Wai, S. R. Knezevich, T. Lucas, B. Jansen, R. J. Kay, and P. H. B. Sorensen, "The ETV6-NTRK3 gene fusion encodes a chimeric protein tyrosine kinase that transforms NIH3T3 cells," Oncogene, vol. 19, no. 7, pp. 906-915, 2000.

[11] S. R. Knezevich, M. J. Garnett, T. J. Pysher, J. B. Beckwith, P. E. Grundy, and P. H. B. Sorensen, "ETV6-NTRK3 gene fusions and trisomy 11 establish a histogenetic link between mesoblastic nephroma and congenital fibrosarcoma," Cancer Research, vol. 58, no. 22, pp. 5046-5048, 1998.

[12] J. M. Bourgeois, S. R. Knezevich, J. A. Mathers, and P. H. B. Sorensen, "Molecular detection of the ETV6-NTRK3 gene fusion differentiates congenital fibrosarcoma from other childhood spindle cell tumors," The American Journal of Surgical Pathology, vol. 24, no. 7, pp. 937-946, 2000.

[13] J. A. Bishop, R. Yonescu, D. A. S. Batista, W. H. Westra, and S. Z. Ali, "Cytopathologic features of mammary analogue secretory carcinoma," Cancer Cytopathology, vol. 121, no. 5, pp. 228-233, 2013. 


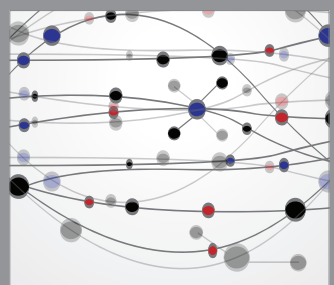

The Scientific World Journal
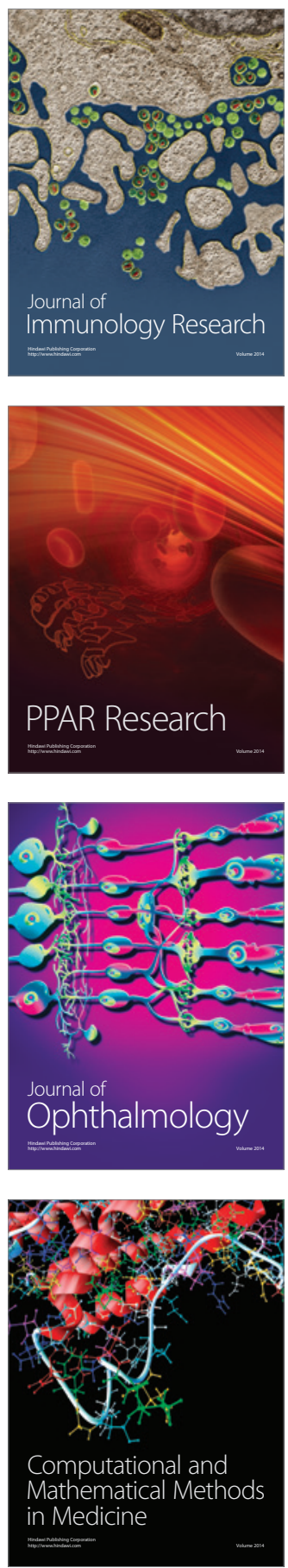

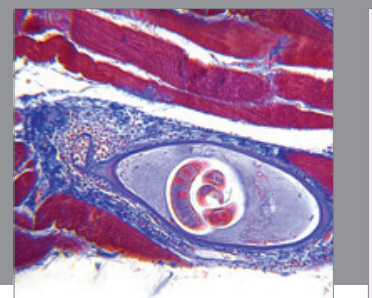

Gastroenterology

Research and Practice
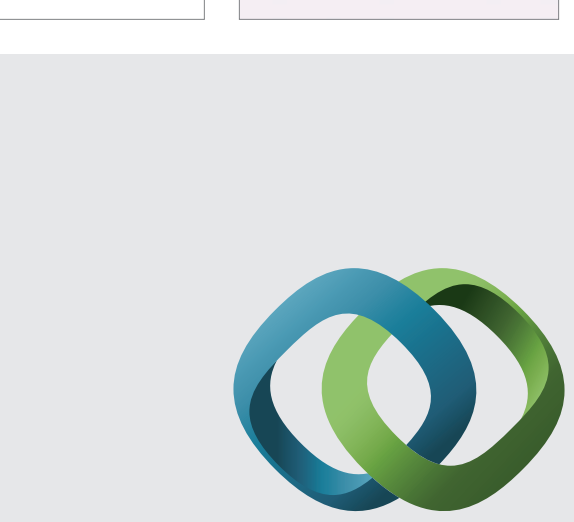

\section{Hindawi}

Submit your manuscripts at

http://www.hindawi.com
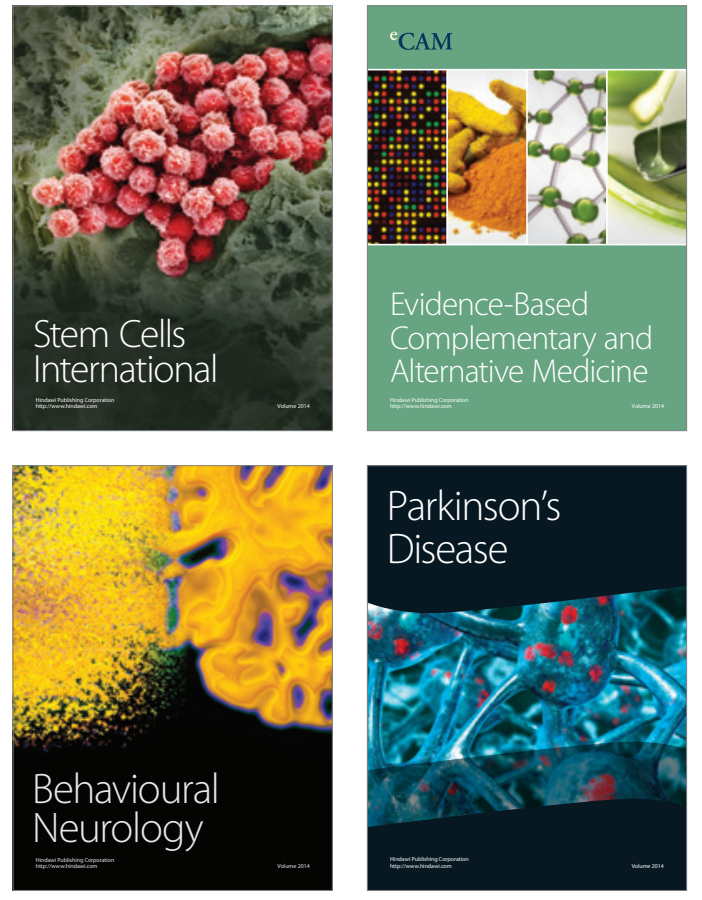
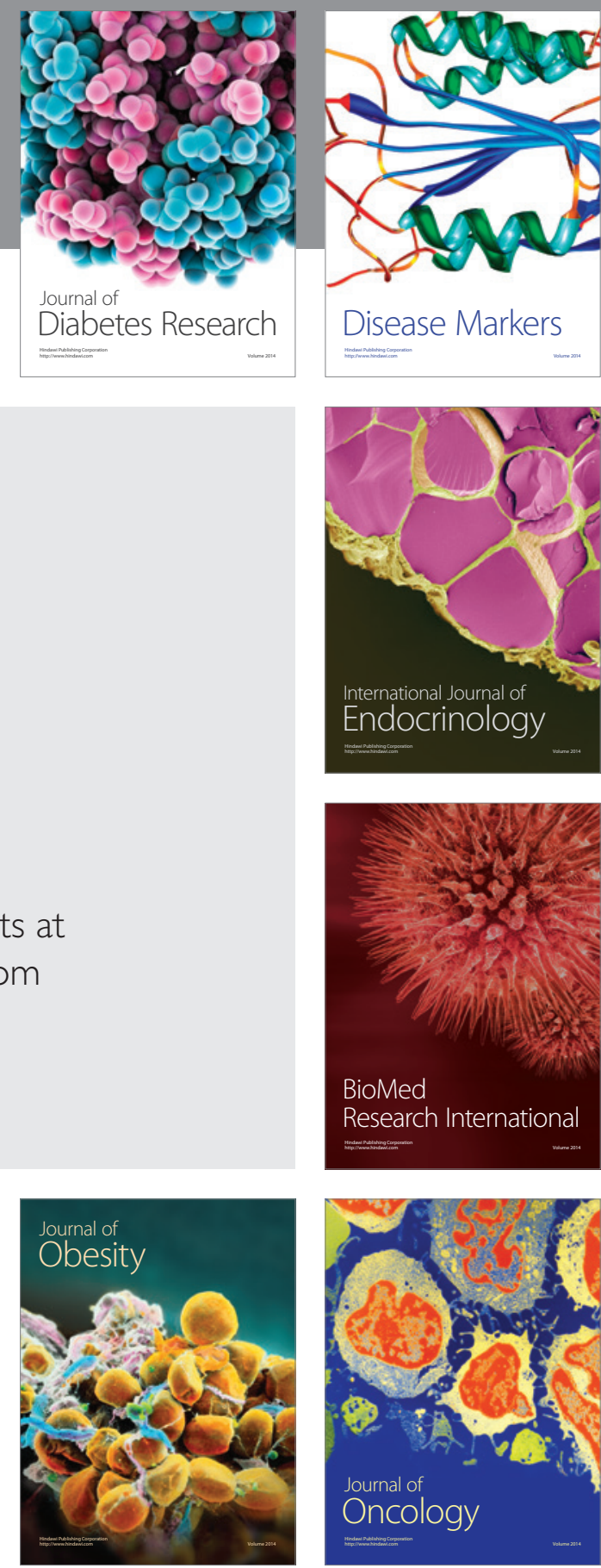

Disease Markers
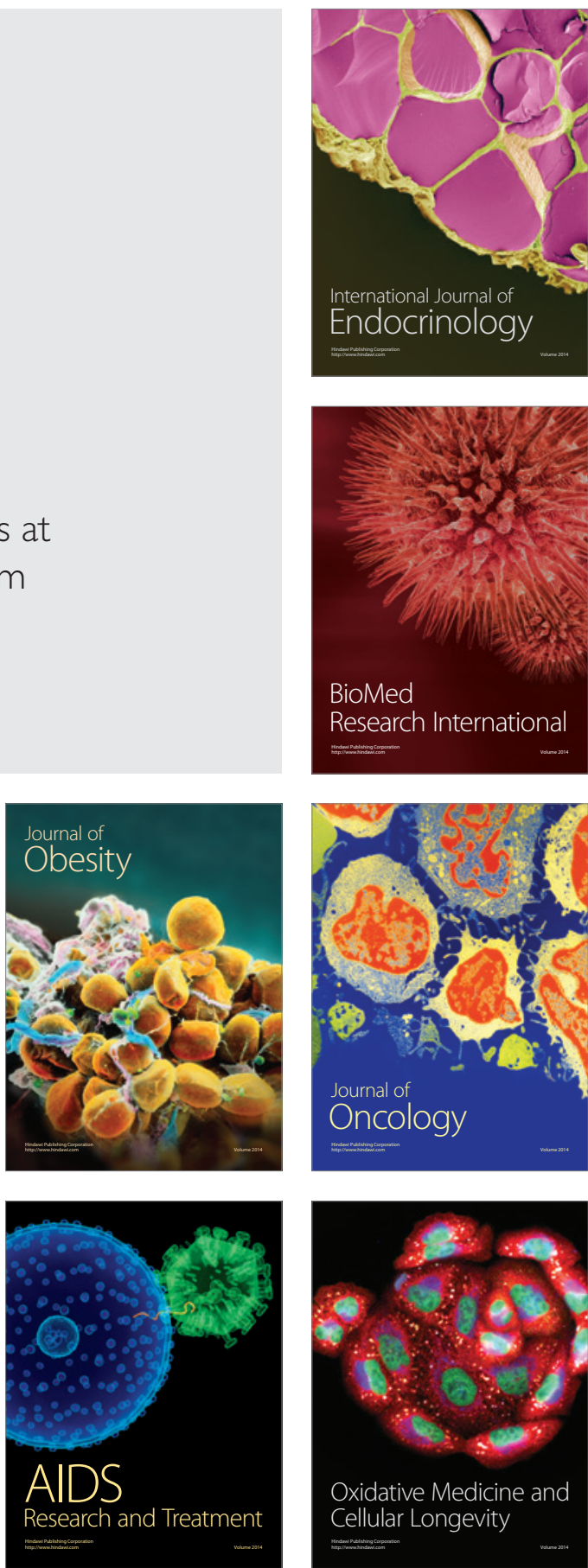